[Vicino Oriente XIX (2015), pp. 39-50]

\title{
UNA TESTA DI SILENO IN BRONZO DA MOZIA
}

\author{
Federica Spagnoli - Sapienza Università di Roma
}

The occasional (and fortuitous) discovery of a bronze head of a silenus, occurred during the $X X X V$ season of excavations carried out by Rome «La Sapienza» University Expedition to Motya, gives the cue to shine a light on the reception and the re-interpretation of Greek themes and iconographies in a period of deep changes on the culture of the island.

Keywords: silenus; bronze toreutics; weight; Greek iconography; Motya

\section{INTRODUZIONE}

Questo articolo trae spunto dal rinvenimento, durante la XXXV campagna di scavi a Mozia della Missione archeologica della Sapienza ${ }^{1}$, di un reperto di bronzo che offre l'occasione per una riflessione sulla circolazione di iconografie ellenizzanti a Mozia e sulla valenza semantica che è ad esse attribuita.

\section{LA TESTA IN BRONZO MAC. 15.500}

L’oggetto (fig. 1), occasionalmente rinvenuto al di fuori del suo contesto originario nei pressi della Zona Abitato Centrale ${ }^{2}$, raffigura un volto umano barbuto (fig. 2) ${ }^{3}$. Ha una forma ovale di $4,5 \times 2,7 \mathrm{~cm}$, lo spessore massimo, misurato in corrispondenza della fronte, è di $1,3 \mathrm{~cm}$. La parte posteriore è liscia e piatta, la parte inferiore è convessa verso l'alto in corrispondenza dell'assottigliarsi dello spessore della barba (fig. 3). Nella parte anteriore, i capelli, divisi al centro, lasciano scoperta la sommità (calva) della testa, scendono lungo i lati della fronte e del viso e si ricongiungono alla barba. Questa termina in una forma arcuata e regolare. I dettagli della capigliatura, della barba e delle folte sopracciglia sono resi con sottili e accurate incisioni ondulate parallele. La fronte prominente è leggermente corrugata mentre le sopracciglia, inarcate e unite al centro, incorniciano i grandi occhi dalle palpebre molto rilevate e dai globi oculari sporgenti, il naso camuso e gli zigomi pronunciati. I baffi sono leggermente rilevati rispetto alla barba e lasciano scoperte le labbra carnose atteggiate nel sorriso arcaico. Il dettaglio delle orecchie mancanti ${ }^{4}$ non

1 Desidero ringraziare il direttore della Missione archeologica a Mozia, Professor Lorenzo Nigro, per avermi affidato la pubblicazione del reperto oggetto di questo articolo. Un sentito ringraziamento alla Professoressa Laura M. Michetti per i preziosi consigli e i suggerimenti bibliografici concernenti i confronti con il repertorio etrusco. Ringrazio, infine, la Dott.ssa Diana Balboa Lagunero che ha ritrovato il reperto.

2 L'area, già individuata da Giuseppe Whitaker nei primi decenni del secolo scorso (Whitaker 1921, 127; Tusa 1969, 10, nota 4), e successivamente scavata in più campagne da Vincenzo Tusa dal 1968 (Tusa 1969, 7-34; 1970, 51-62), è localizzata nel settore centro-settentrionale dell'isola, lungo una direttrice viaria NE-SO, sulla quale si affaccia un complesso edilizio all'interno del quale era un edificio a destinazione cultuale. L'area, occupata dopo la distruzione della città del 397/6 a.C., ha restituito reperti databili per lo più tra il IV e il III secolo a.C.; tuttavia, sono presenti anche materiali di VI e V secolo a.C. a testimonianza di un'occupazione più antica dell'area (Tusa 1970, 53, tavv. XXXIII:1, 59; XXXVI:1, 60; XXXVII:1; Lisella 2004, 64, con bibliografia precedente).

3 Il disegno è stato realizzato dalla Sig.ra Anna Sumislawska, che sentitamente ringrazio.

4 Sulla testa sono tuttavia visibili, seppur parzialmente nascoste dalle incrostazioni, delle tracce circolari che potrebbero segnalare la base delle orecchie (perdute). Ringrazio per questa nota la Dott.ssa Daria Montanari. 
consente di identificare con certezza il personaggio raffigurato: tuttavia, il tipo di capigliatura, la barba, e soprattutto l'espressione beffarda del volto, avvicinano questa figura all'iconografia del sileno barbuto di ascendenza ionica ${ }^{5}$. Riguardo alla tecnica di manifattura, per il peso del pezzo ${ }^{6}$ si deve escludere la tecnica a cera persa a favore di una procedura a fusione piena, con profilatura a freddo mediante incisione a cesello dei particolari.

L'accurata fattura, la bellezza del disegno e lo stato di conservazione rendono questo bronzetto di straordinario interesse, specie se si considera che esso potrebbe provenire da una delle aree sacre minori di Mozia.

\section{CONFRONTI ICONOGRAFICI}

Come quasi sempre accade quando ci si confronta con i reperti moziesi, lo studio dei temi e delle iconografie, specialmente quelle estranee alla matrice orientale che caratterizza il background culturale dell'isola, pone in evidenza la profonda complessità del contesto culturale in cui ci si muove. Per questo motivo, anche nell'analisi del bronzetto è necessario, ancora una volta, tenere in considerazione una doppia prospettiva di lettura: la prospettiva dell'artigiano - probabilmente greco - che ha prodotto l'oggetto, che attinge al proprio bagaglio culturale di conoscenze, di temi e di iconografie, e quella dell'acquirente e fruitore, partecipe di una dimensione ideologica estranea al milieu di origine del prodotto ${ }^{7}$.

\subsection{Levante}

La testa in bronzo da Mozia appartiene ad un tipo iconografico ben noto sia nella pittura vascolare sia nella bronzistica e nella statuaria, nel mondo greco ma anche al di fuori di esso, sia in Occidente ${ }^{8}$ che nel Levante ${ }^{9}$. Con particolare riferimento a questa regione, si cita come possibile confronto un peso fenicio in piombo proveniente da Amrit, configurato a testa di sileno (fig. 4) ${ }^{10}$. Il soggetto rappresentato è diverso dall'esemplare moziese per la capigliatura folta che copre parzialmente la fronte. La fattura è meno accurata nella resa dei particolari di occhi e barba. Come il bronzetto moziese, il sileno di Amrit è privo delle orecchie: nonostante l'assenza di questo elemento, le caratteristiche del volto lo avvicinano all’iconografia più tradizionale del sileno. Il peso di piombo di Amrit è stato datato al VI

5 Higgins 1954, 73-74, nn. 159-165, tav. 31; Moscati 1987, 12; Bini - Caramella - Buccioli 1995, 96.

6 L'oggetto pesa circa 62,4 gr. Facendo riferimento al sistema ponderale calcidese, ampiamente diffuso nel Mediterraneo occidentale alla metà del I millennio a.C., il peso corrisponde a circa 12 volte il piede calcidese di 5,40 g. Un'ampia disamina dei sistemi ponderali utilizzati a Mozia tra i V e il IV secolo a.C. in Ascalone 2004, con particolare riferimento a p. 439, nota 2.

7 Isler-Kerényi 2002, 69-70; 2003, 39-41. Questa ambivalenza delle prospettive interpretative, che è una questione aperta anche in altre aree di penetrazione della cultura greca, è stata da ultimo evidenziata per il mondo punico in Nigro 2015, 229.

8 La bibliografia delle attestazioni di questo tipo iconografico in Occidente è amplissima; riferimenti al mondo italico e magno greco di seguito alle note 12, 14-16; per il mondo punico si cita, a titolo esemplificativo, il recente lavoro di P. De Vita (2009), con ampia bibliografia precedente.

$9 \quad$ Fantalkin 2006 con bibliografia precedente.

10 I pesi cefalomorfi non sono insoliti nel mondo fenicio: sono infatti attestati anche Ugarit e, in ambito coloniale, a Ibiza: Elayi - Elayi 1997, 189-190, n. 451, tav. XXXVI. 
secolo a.C., sulla base dello stile che risente dell'influenza della plastica di ispirazione greco-cipriota ${ }^{11}$.

\subsection{Grecia ed Etruria}

L'iconografia del sileno è molto comune nel mondo greco, magno greco ed etrusco italico non solo nella toreutica, ma anche nella coroplastica, a tuttotondo e architettonica.

Naturalmente ampia attestazione di questo soggetto si trova nella pittura vascolare attica e siceliota tra il VI e il IV secolo a.C. In particolare deve essere citato, tra i numerosissimi esempi che si potrebbero portare a confronto, uno stamnos attico a figure rosse, datato tra il 475 e il 425 a.C., rinvenuto a Chiusi e oggi esposto al British Museum: sul vaso sono rappresentati dei sileni che hanno un volto simile a quello del reperto moziese, caratterizzato da calvizie, naso camuso, barba folta e compatta ${ }^{12}$.

Dal mondo etrusco provengono i confronti iconografici più vicini al nostro bronzetto ${ }^{13}$. Teste barbute e figure di sileni, infatti, sono soggetti molto comuni nella bronzistica etrusca di VI e V secolo a.C. sia come appliques che come figurine a tuttotondo. Esemplare è il caso del thymiaterion in bronzo di Magonza della Collezione L. Fould, in cui, nella parte inferiore, sono raffigurati due sileni itifallici in corsa, i cui volti sono confrontabili con l'esemplare moziese, e quattro appliques a protome di Acheloo, avvicinabili al nostro esemplare per forma e tecnica di lavorazione ${ }^{14}$. Appliques a testa di sileno sono frequenti anche come elementi decorativi degli elmi in bronzo, cui erano fissati mediante perni posti nella parte posteriore ${ }^{15}$. Un altro confronto, sempre da area etrusca, è in una brocca con ansa "à la crosse" desinente in una maschera di sileno ${ }^{16}$.

I confronti citati sono databili tra la fine del VI secolo e il primo trentennio del V secolo a.C.

\subsection{Sicilia e Magna Grecia}

L'iconografia del sileno si afferma in Sicilia a partire dalla seconda metà del VI secolo a.C.: è in questo periodo che si diffonde l'uso di decorare il bordo del tetto degli edifici sacri con antefisse raffiguranti delle teste di sileno, forse derivazione della consuetudine degli attori dei drammi satireschi vincitori negli agoni drammatici di offrire le maschere di

11 Elayi - Elayi 1997, 150. Il peso misura 3,56 × 1,8 $\times 1,3 \mathrm{~cm}$, e pesa 45,10 g. Peso a forma di testa umana, con capelli con frangia che scende sulla fronte, viso dai tratti pieni e labbra carnose. Le sopracciglia, arcuate e leggermente prominenti, sovrastano i grandi occhi dall'espressione seria. La parte posteriore del peso è piatta.

12 Corpus Vasorum Antiquorum: London British Museum 3, III.Ic.9, pl. (187) 22:2 A-C.

13 Alcuni tipi iconografici (tra i quali si annovera anche quello del sileno, insieme, ad esempio, alle rappresentazioni di Demetra con polos schiacciato) testimoniano una "sintonia" tra produzioni artigianali, inserita in una comunanza di gusti tra ambiente greco, etrusco e punico, tra l'età arcaica e il III secolo a.C.: Michetti 2007, 335. Per uno sguardo d'insieme sulla diffusione, in ambiente etrusco, di pratiche religiose legate al mondo dionisiaco e sui modi in cui queste pratiche si riflettono nell'utilizzo di certi tipi iconografici, si veda Colonna 1991, in particolare p. 118, nota 15.

14 Naso 2003, 95-97, n. 146, tavv. 51-53.

15 Bini - Caramella - Buccioli 1995, 487-488, n. 96, tav. CII:1 (s.n. inv.).

16 Bini - Caramella - Buccioli 1995, 45-46, n. 79, tavv. XXXI:4; CXVII:1 (inv. 5688). Nella maschera è possibile ravvedere affinità formali con l'antefissa silenica del Tempio Grande in Contrada Vignale (Falerii): Andrén 1940, 95, I:3, t.29:102:VI-V secolo a.C. 
scena. Queste terrecotte architettoniche erano dipinte a colori vivaci, e riproducevano la policromia delle maschere teatrali ${ }^{17}$. L'iconografia più arcaica, di fine VI secolo a.C., attestata a $\operatorname{Naxos}^{18}$ e in altri centri della Sicilia Orientale, quali Siracusa ${ }^{19}$ e Camarina ${ }^{20}$, rappresenta un sileno dal volto composto, quasi ieratico: barba regolare, folta capigliatura che copre l'alta fronte distesa, tratti somatici di stile severo ma senza sorriso arcaico ${ }^{21}$. Successivamente (V-IV secolo a.C.) si afferma un altro tipo iconografico, documentato a Naxos $^{22}$ e a Gela ${ }^{23}$, in cui viene accentuata la natura ferina e bestiale del personaggio, con calvizie e barba folta e disordinata, fronte corrugata, naso camuso, labbra socchiuse in un ghigno con denti in vista ${ }^{24}$. Il bronzetto moziese ha elementi in comune con entrambe le tipologie e si pone dunque in una posizione intermedia in cui l'aspetto ferino del personaggio è appena accennato. In Italia meridionale, antefisse sileniche sono attestate a Taranto ${ }^{25}$, in un arco cronologico piuttosto ampio che include anche il periodo ellenistico, e a Medma, dove sono state rinvenute delle antefisse relative ad un edificio sacro del terzo quarto del VI secolo a.C. ${ }^{26}$ in cui sono rappresentati sileni in corsa. Rimanendo nello stesso ambito culturale, nella Casa dei Leoni a Locri è stata rinvenuta una matrice in terracotta di sileno barbuto che, pur essendo cronologicamente distante (IV secolo a.C.), è confrontabile con il reperto moziese ${ }^{27}$.

\subsection{Mozia e la Sicilia Occidentale}

Le indagini archeologiche effettuate a Mozia negli ultimi decenni dalla Missione archeologica dell’Università di Roma «La Sapienza», hanno portato alla luce alcune statuette, principalmente teste, e matrici raffiguranti questo soggetto. Come nei centri punici della Sicilia Occidentale, anche a Mozia sono numerosi i vasi di importazione greca, in particolare crateri e coppe a figure rosse, in cui sileni e satiri sono rappresentati come attori principali o come personaggi secondari ${ }^{28}$. Tuttavia, nonostante i numerosi confronti iconografici ravvisabili in altre classi di materiali, la testa bronzea in oggetto costituisce, allo stato attuale delle attestazioni, almeno nell'ambito moziese, un unicum nel suo genere.

17 Nel dramma satiresco di Eschilo Theōroi ê Isthmiastai la maschera, o prosōpon, è detta kalligraphtos, cioè imbellettata, truccata: Marconi 2005, 78, nota 2.

18 Pelagatti 1965, nn. 1-14, tavv. XXXI:1-3, XXXII:1-4, XXXIV:1-3; 1980-1981, 704, nota 37.

19 Pelagatti 1965, 84, n. 14, tav. XXXII:1; 2003, 518

20 Pelagatti 2003, 517, figg. 1-2.

21 Questo particolare ha indotto alcuni studiosi ad abbassare la datazione al 490-480 a.C.: Orlandini 1954, tav. LXXXI: 1-3; Winter 1903, 279.

22 Pelagatti 1965, nn. 15-32, tavv. XXXIV:4-7; XXXV:1-2, 4; XXXVI: 2; XXXVII:1-2, 4.

23 Il tipo c.d. “di via Apollo”. Della consistente bibliografia, si citano: Orlandini 1954, tavv. LXXXV-LXXXIX; 1956, tav. XVII; Orlandini - Adamesteanu 1956, figg. 1-8; da ultima Pelagatti 2003, 518-519. Per l'attestazione del tipo in altri centri sicelioti: Pelagatti 2003, 529, nota 33 (Sabucina); 519, fig. 6 (Camarina).

24 Questo tipo di iconografia si afferma in Sicilia tra il 460 e il 450 a.C.: Pelagatti 2003, 518.

25 Winter 1903, 287.

26 Orsi 1913, 67-68, fig. 73.

27 Barra Bagnasco 1992, 288, n. 287, tav. LXXXIV; proposte di interpretazione della valenza religiosa delle figure di satiri e sileni rinvenuti nella Casa dei Leoni: 284, nota 107.

28 Nigro - Spagnoli 2012, 53, MC.11.4512/2 tav. V; Nigro 2007, 76, MD.03.1056/18, tav. LXVIII; MD.03.1056/61, tav. LXIX; 2009, MF.05.1287/4, tav. LXXXV, figg. 3.84-3.85. Ulteriore bibliografia in Chiarenza 2015 
genere. Il successo che l'iconografia riscuote anche in altri centri punici del Mediterraneo centrale e occidentale dal V secolo a.C. è testimoniata, ad esempio, dalla produzione di maschere sileniche ispirate ai modelli greco-arcaici in Sardegna ${ }^{29}$.

\section{PROPOSTE INTERPRETATIVE}

In assenza di un contesto di rinvenimento stratigraficamente affidabile, l'inquadramento cronologico proposto si basa esclusivamente su fattori stilistici e iconografici, rispetto ai confronti sopra citati. La testa presenta delle caratteristiche proprie della plastica greca di VI secolo a.C.: la barba è unita ai capelli e termina con un bordo semicircolare regolare, le labbra sono chiuse nel sorriso arcaico, e anche l'espressione degli occhi, seppur beffarda, non turba quel senso di compostezza emanato dal volto ${ }^{30}$. La calvizie che lascia scoperta la fronte corrugata, invece, ricollega l'esemplare moziese alla rappresentazione dei sileni nel periodo classico, principalmente quelle sui vasi attici a figure rosse e in alcune antefisse sileniche siceliote della prima metà del V secolo a.C., senza però conferire al volto l'aspetto bestiale e scomposto di queste figure ${ }^{31}$. Queste osservazioni fanno protendere verso una datazione del reperto in un periodo compreso tra la fine del VI e l'inizio del V secolo a.C.

Sulla funzione di tale reperto si rimane nel campo delle ipotesi. La testa, che, ricordiamo, non è a tuttotondo, ma ha la parte posteriore liscia, potrebbe essere l'applique di un arredo, bronzeo o di altro materiale, cui originariamente sarebbe stata incastrata, non essendo presenti, infatti, perni o ganci. Per questo motivo l'ipotesi che si tratti di un peso in bronzo conformato a testa di sileno, pur in assenza di confronti puntuali in ambito punico, rimane la più plausibile.

Per quanto riguarda il soggetto rappresentato, occorre considerare che il valore semantico attribuito dal fruitore moziese all'immaginario di matrice ellenica è anche legato alle modalità di recezione e di assimilazione di modelli iconografici e concettuali provenienti dal mondo greco. La documentazione iconografica del soggetto in questione, infatti, è attestata in un momento di profonda trasformazione che vede l'elemento greco affermarsi con forza nel tessuto culturale di impronta orientale che aveva caratterizzato la Mozia delle origini. In questa ottica si rende necessaria quella doppia prospettiva di interpretazione dei manufatti importati, attraverso la quale proveremo ad analizzare il bronzetto moziese.

\subsection{La prospettiva greca: satiri e sileni, questioni iconologiche}

Assenti nella Teogonia, i sileni, così come i satiri, godettero a lungo di uno status mitologico particolare, essendo privi di una genealogia definita che giustificasse o spiegasse il motivo del loro aspetto ibrido, che univa la forma umana e quella caprina ed equina. L'unica eccezione è costituita da Sileno, precettore di Dioniso. Nella drammaturgia

29 Ciasca 1988, 363-365; Orsingher 2014, 147-148.

30 Gli stessi tratti stilistici che si riscontrano anche nei volti delle figure metopali dei templi della vicina Selinunte: Tusa 1984, 135-137. Le produzioni coroplastiche selinuntine di età arcaica recepiscono influenze stilistiche di origini diverse (ionica, corinzia, attica) rielaborandole in forme eclettiche che si muovono nel solco di una specificità stilistica originale: Pautasso 2012, 115-116, con bibliografia precedente.

31 I tempi e di modi di questa trasformazione, che avviene nel primo quarto del V secolo a.C., sono tuttora oggetto di discussione tra gli studiosi; si rimanda pertanto a discussione a Marconi 2005, in particolare 84-85. 
essi sono protagonisti dei drammi satireschi, nei quali il coro era formato da attori travestiti da satiri. La notizia che i satiri potessero essere figli di Sileno scaturisce proprio dal fatto che ne "Il Ciclope" di Euripide il satiro più anziano, chiamato Sileno, esercita un'autorità paterna sui compagni. Dei due nomi utilizzati dalle fonti "sileno" sembra essere più antico $^{32}$, mentre "satiro" viene preferito già in Euripide ${ }^{33}$. In Platone i due nomi sono intercambiabili ${ }^{34}$, ma nel corso del V secolo a.C., proprio grazie ai drammi satireschi, si afferma una distinzione, basata sull'età, tra sileni anziani (barbuti) e satiri giovani (imberbi) ${ }^{35}$.

Il legame tra sileni e Dioniso, tuttavia, non è all'origine di queste figure mitologiche. Inizialmente, infatti, questi erano personaggi del tutto indipendenti dal dio, più vicini ai demoni della natura, benefattori e protettori, estranei al mondo dionisiaco. La religione dionisiaca adotterà progressivamente quelle figure mitologiche legate alle sorgenti, alle acque e alle foreste, come i sileni e i satiri, ma in grado di rappresentare anche l'aspetto istintivo e bestiale della natura umana. A questo ultimo aspetto si rifà l'immagine letteraria trasmessa dal dramma satiresco: quella di creature dagli istinti primordiali, che si collocano agli antipodi della società civilizzata che desta in loro sgomento e stupore attonito ${ }^{36}$.

\subsection{La prospettiva punica: gli elementi di mediazione}

Il significato che in ambiente punico moziese viene attribuito ai sileni si discosta da quello greco originario, ed è legato alla parziale trasformazione che essi subirono nel corso del VI secolo a.C., quando gli intensi contatti tra il mondo greco e quello egiziano favorirono dei fenomeni sincretici che investono queste figure ${ }^{37}$. In questo periodo si verifica, infatti, la sovrapposizione tra la figura del sileno e quella di Bes, soprattutto in ambito coloniale ${ }^{38}$. L'associazione tra queste figure mitologiche non si limita all'aspetto iconografico, ma riguarda anche aspetti più propriamente religiosi: sovrapponendosi a Bes, divinità protettrice, legata al mondo dell'infanzia e femminile, alla sfera della fecondità, della rigenerazione e delle fasi di passaggio dell'esistenza umana, anche i sileni assumeranno queste prerogative.

I fenomeni sincretici interessano proprio questo tipo di religiosità popolare che, nel corso del VI secolo a.C., si sovrappone alla sfera religiosa dionisiaca. L’influenza della religione egiziana investe anche la figura di Dioniso che, già accostato a Kore-Persephone e Demetra nei misteri Eleusini nel suo carattere ctonio e infero, arriva a essere assimilato, nel corso del V secolo a.C., a Osiride.

\footnotetext{
Inno ad Afrodite, v. 262

Euripides, Cyclops, v. 100; Bacchae, v. 13.

Platone Symposium 215b, 216c.

Pausania, I 23, 5.

36 Seaford 1976, 212; Lissarrague 1993, 219. Il dramma satiresco era un modo per esplorare la cultura attraverso i suoi antipodi, in un processo di straniamento e ricomposizione. Agli antipodi sono appunto i satiri, veri e propri rappresentanti dello stato di natura: Lissarrague 1990, 235 passim.

37 Capriotti Vittozzi 2003, 145-149; Sannibale 2008, 183-184.

38 Capriotti Vittozzi 2011, in particolare 113-114.
} 


\section{CONCLUSIONI}

Figure sileniche a Mozia, insieme ad altri oggetti che afferiscono anche indirettamente alla sfera dionisiaca ${ }^{39}$, sono attestate a partire dal primo venticinquennio del V secolo a.C., nel periodo in cui l'ellenizzazione della cultura locale, già in atto dalla seconda metà del VI secolo a.C., subisce una notevole accelerazione, fino ad arrivare ad essere pressoché completa alla fine del secolo ${ }^{40}$. Nel caso specifico, questa presenza indica più che la forza di penetrazione di iconografie greche, il ruolo ancora determinante che il sostrato egiziano ricopre nella cultura moziese, anche in un momento di grandi cambiamenti. Per entrambe le sfere, quella religiosa e quella iconografica, infatti, la recezione e la successiva assimilazione, da parte dei fruitori punici, di modelli culturali e formali estranei (come sono appunto i sileni), non avviene in maniera diretta, ma è probabilmente favorita dalla mediazione della cultura egiziana che, profondamente radicata nella matrice culturale fenicia, rimane ancora tra il VI e la prima metà del V secolo a.C., una componente costante e vitale, fornendo, come in questo caso, una chiave di lettura comune alla cultura greca e a quella semitica.

Concludendo, il rinvenimento della testa in bronzo suggerisce un'ultima considerazione di cui è necessario tenere conto quando si parla di ricezione e assimilazione di modelli esterni $^{41}$ : Mozia, nel V secolo a.C. importa oggetti di lusso che hanno un valore in quanto beni pregiati e di altissima qualità artistica, ed è verosimile pensare che potrebbero anche non avere avuto per l'acquirente un valore simbolico e rappresentativo ${ }^{42}$. Questi oggetti sono le tessere di un puzzle ancora incompleto, che l'archeologo deve ricomporre, che restituisce l'immagine, seppur parziale, di una società raffinata e cosmopolita, che vuole essere pervasa, che vuole nutrirsi della cultura greca, forse solo per una questione di prestigio, forse per puro edonismo oppure, più verosimilmente, per una sincera attitudine ad accogliere, ad assimilare e a riformulare in chiave originale stimoli provenienti da culture altre, un'attitudine dettata da quel carattere internazionale ed ecumenico che è tipico dei Fenici di tutte le epoche.

\section{BIBLIOGRAFIA}

ANDRÉN, A.

1940 Architectural terracottas from Etrusco-Italic Temples, Lund - Leipzig 1940.

ASCALONE. E.

2004 Nota su un peso in piombo da Mozia: L. Nigro (a cura di), Mozia - X. Zona C. Il Kothon. Zona D. Le pendici occidentali dell'Acropoli. Zona F. La porta ovest. Rapporto preliminare della XXII campagna di scavi - 2002 condotta congiuntamente con il Servizio Beni Archeologici della Soprintendenza Regionale per i Beni Culturali e Ambientali di Trapani (Quaderni di Archeologia Fenicio-Punica I), Roma 2004, pp. 439-444.

39 Citiamo, altre i reperti menzionati prima, nota 28, anche un frammento di un mortarium in marmo di I-II secolo d.C. (n. inv. 6085 Coll. Whitaker) con raffigurazione di delfini in doppia coppia ed elementi vegetali, proveniente da Mozia o da un centro vicino: Olivieri 2009, 336, nota 17.

40 Spagnoli 2013, 160; 2014, 117-118; Nigro 2015, 222.

41 Spagnoli 2012, 307; Orsingher 2013, 696.

42 Michetti 2007, in particolare 331-333; Nigro 2009, 708; per gli oggetti di lusso in metallo: Talone $2016,142$. 
BARRA BAGNASCO, M.

1992 Locri Epizefiri IV. Lo scavo di Marasà sud. Il Sacello tardo-arcaico e la Casa dei Leoni (Studi e Materiali di Archeologia 4), Firenze 1992.

Bini, M.P. - CARAMELLA, G. - BuCCIOLI, S.

1995 I bronzi etruschi e romani (Materiali del Museo Archeologico Nazionale di Tarquinia XIII), Roma 1995.

CAPRIOTTI VITTOZZI, G.

2003 Il fanciullo, il nano e la scimmia: figure "grottesche" e religiosità popolare tra greci ed egizi: F. Costabile (a cura di), Polis 1. Studi Interdisciplinari sul Mondo Antico, Roma 2003, pp. 141-154.

2011 Elementi di tradizione egizia nella documentazione di Locri: M. INTRIERI - S. RIBICHINI (a cura di), Fenici e Italici, Cartagine e la Magna Grecia. Popoli a contatto, culture a confronto. Atti del Convegno Internazionale Cosenza, 27-28 Maggio 2008 (Rivista di Studi Fenici 36/1-2), Pisa-Roma 2011, pp. 109-128.

CHIARENZA, N.

2015 Una matrice per terrecotte con sileno dall'area sacra del Kothon a Mozia: Vicino Oriente XIX (2015), pp. 51-64.

CIASCA, A.

1988 Le protomi e le maschere: S. Moscati (a cura di), I Fenici. Catalogo della mostra, Palazzo Grassi, Venezia, 6 marzo - 6 novembre 1988, Milano 1988, pp. 354-369.

COLONNA, G.

1991 Riflessioni sul dionisismo in Etruria: F. BERTI (a cura di), Dionysos. Mito e mistero. Atti del Convegno internazionale, Comacchio 1989, Ferrara 1991, pp. 117-155.

ELAYI, J. - ELAYI, A.G.

1997 Recherches sur les poids Phéniciens (Suppl. n. 5 à Transeuphratène), Paris 1997.

FANTALKIN, A.

2006 Identity in the Making. A. VILLING - U. SCHLOTZHAUER (eds.), Naukratis: Greek Diversity in Egypt. Studies on East Greek Pottery and Exchange in the Eastern Mediterranean (British Museum Research Publication 162). London 2006, pp. 199-208.

HIGGINS, R.A.

1954 Catalogue of the Terracottas in the Department of Greek and Roman Antiquities, British Museum. Vol. I, Greek: 730-330 B.C., London 1954.

ISLER-KERÉNYI, C.

2002 Un cratere polignoteo fra Atene e Spina: Numismatica e Antichità Classiche 31 (2002), pp. 69-88.

LISELLA, A.R.

2004 L’Abitato Centrale: L. Nigro - G. Rossoni (a cura di), «La Sapienza» a Mozia. Quarant'anni di ricerca archeologica, 1964-2004. Catalogo della Mostra, Università di Roma "La Sapienza", Facoltà di Scienze Umanistiche, Museo dell'Arte Classica, 27 febbraio-18 maggio 2004 (Quaderni di archeologica Fenicio-Punica/Colour Monograph 01), Roma 2004, pp. 62-65.

LISSARRAGUE, F.

1990 Why Satyrs are Good to Represent: J.J. WinKLER - F. ZeiTLin (eds.), Nothing to Do with Dionysos? Athenian Drama in its Social Context, Princeton 1990, pp. 228-236.

1993 On the Wildness of Satyrs: Th.H. CARPENTER - Ch.A. FARAONE (eds.), Masks of Dionysus, Ithaca - New York 1993, pp. 207-220.

MARCONI, C.

2005 I Theōroi di Eschilo e le antefisse sileniche siceliote: Sicilia antiqua: International Journal of Archaeology 2 (2005), pp. 75-93. 
MiCHETTI, L.M.

2007 Scambi e interferenze culturali tra ambiente etrusco-italico e mondo punico: alcuni esempi nell'artigianato artistico di età recente (IV-III sec. a.C.): Etruschi, Greci, Fenici e Cartaginesi nel Mediterraneo centrale. XIV Convegno internazionale di Studi sulla storia e l'archeologia dell'Etruria, Orvieto 2006 (Annali per la Fondazione del Museo "C. Faina” XIV), Roma 2007, pp. 325-363.

MosCati, S.

1987 Iocalia punica. La collezione del Museo Nazionale G.A. Sanna di Sassari: Atti della Accademia Nazionale dei Lincei. Classe di Scienze morali, storiche e filologiche.

NASO, A

2003 I bronzi etruschi e italici del Römisch-Germanisches Zentralmuseum (Verlag des Römisch-Germanischen Zentralmuseums), Mainz am Rhein 2003.

NigRO, L.

2007 Mozia - XII. Zona D. La "Casa del sacello domestico", il "Basamento meridionale" e il Sondaggio stratigrafico I. Rapporto preliminare delle campagne di scavi XXIII e XXIV (2003-2004) condotte congiuntamente con il Servizio Beni Archeologici della Soprintendenza Regionale per i Beni Culturali e Ambientali di Trapani (Quaderni di Archeologia Fenicio-Punica III), Roma 2007.

2009 Offerte e depositi votivi nel Santuario C3 del Kothon di Mozia nel IV secolo a.C.: S. FortunELli - C. MASSERIA (a cura di), Ceramica attica da santuari della Grecia, della Ionia e dell'Italia (Atti Convegno Internazionale Perugia 14-17 marzo 2007), Venosa 2009, pp. 703-719.

2011 Mozia - XIII. Zona F. La Porta Ovest e la Fortezza Occidentale. Rapporto preliminare delle campagne di scavi XXIII - XXVII (2003-2007) condotte congiuntamente con il Servizio Beni Archeologici della Soprintendenza Regionale per i Beni Culturali e Ambientali di Trapani (Quaderni di Archeologia Fenicio-Punica VI), Roma 2011.

2015 Mozia tra VI e V secolo a.C. Monumentalizzazione e organizzazione socio-politica: un nuovo modello: M.P. BAgLione - L.M. MichetTi (a cura di), Le lamine d'oro a cinquant'anni dalla scoperta. Dati archeologici su Pyrgi e rapporti con altre realtà del Mediterraneo (Scienze dell’Antichità 21.2), Roma 2015, pp. 225-245.

NigRo, L. - SPAGNOLI, F.

2012 Alle sorgenti del Kothon. Il rito a Mozia nell'Area sacra di Baal 'Addir - Poseidon. Lo scavo dei pozzi sacri nel Settore C Sud-Ovest (2006-2011) (Quaderni di Archeologia Fenicio-Punica/Colour Monograph 02), Roma 2012.

OLIVIERI, F.

2009 Le raffigurazioni di delfini: M.L. FAMÀ (a cura di), Il Museo Regionale “A. Pepoli” di ORLANDINI, P. Trapani. Le collezioni archeologiche, Bari 2009, pp. 335-337.

1954 Le nuove antefisse sileniche di Gela ed il loro contributo alla conoscenza della coroplastica siceliota: Archeologia Classica 6 (1954), pp. 251-266.

$1956 \quad$ Altre antefisse sileniche di Gela: Archeologia Classica 8 (1956), p. 47.

ORLANDiNI, P. - ADAMESTEANU, D.

1956 Gela. Ritrovamenti vari: Notizie degli Scavi d'Antichità (1956), pp. 203-401.

ORSI, P.

1913 Rosarno (Medma). Esplorazione di un grande deposito di terrecotte ieratiche: Notizie degli Scavi d'Antichità, Suppl. (1913), pp. 55-144. 
ORSINGHER, A.

2013 The Hellenisation of the Punic World: a view from the Tophet: L. BOMBARDIERI - A. D’Agostino - G. GuARDUCCI - V. ORSI - S. VALENTINI (eds.), SOMA 2012. Identity and Connectivity: Proceedings of the $16^{\text {th }}$ Symposium on Mediterranean Archaeology, Florence, Italy, 1-3 March 2012 (British Archaeological Reports International Series 2581), Oxford 2013, pp. 693-701.

2014 Listen and protect: reconsidering the grinning masks after a recent find from Motya: Vicino Oriente XVIII (2014), pp. 145-171.

PAUTASSO, A.

2012 L'età arcaica. Affermazione e sviluppo delle produzioni coloniali: M. AlBERTOCCHI - A. PAutAsso (eds.), Philotechnia. Studi sulla coroplastica della Sicilia greca, Catania 2012, pp. 113-139.

Pelagatti, P.

$1965 \quad$ Antefisse sileniche siceliote: Cronache d'Archeologia 4 (1965), pp. 79-98.

1980-1981 L'attività della Soprintendenza alle antichità della Sicilia Orientale, 2: Kokalos 26-27 (1980-1981), pp. 694-735.

2003 Antefisse di provenienza camarinese certa o presunta: G. Fiorentini - M. CALTABiANO A. CAlderone (edd.), Archeologia del Mediterraneo. Studi in onore di Ernesto De Miro, Roma 2003, pp. 515-532.

SANNIBALE, $\mathrm{M}$.

2008 La Raccolta Giacinto Guglielmini. Parte II. Bronzi e materiali vari, Roma 2008.

SEAFORD, R.

1976 On the Origins of Satyric Drama: Maia 28 (1976), pp. 209-221.

SPAGNOLI, F.

2012 Un’anforetta dipinta dalla Tomba T.177 di Mozia: Vicino Oriente XVI (2012), pp. 303312.

2013 Demetra a Mozia: evidenze dall'area sacra del Kothon nel V secolo a.C.: Vicino Oriente XVII (2013), pp. 153-165.

2014 Una brocchetta dipinta dal Tempio di Astarte nell’Area Sacra del Kothon a Mozia: Vicino Oriente XVIII (2014), pp. 113-121.

TALONE, M.

2016 Phoenician Metal Jugs: J. JimÉnEz Ávula (ed.), Phoenician Bronzes in Mediterranean (Bibliotheca Archaeologica Hispana 45), Real Academia de la Historia, Madrid 2016, pp. 119-146.

TUSA, V.

1969 Il «centro abitato». Lo scavo del 1968: A. CiAsCA - M.G. GuZzo AmADASI - G. MATTHIAE SCANDONe - B. Olivieri Pugliese - A. Cutroni TusA - V. TusA, Mozia - V. Rapporto preliminare della Missione congiunta con la Soprintendenza alle Antichità della Sicilia Occidentale (Studi Semitici 31), Roma 1969, pp. 7-34.

1970 Il «centro abitato». Lo scavo del 1969: A. CIASCA - M.G. GuZzo AMADASI - S. MosCATI V. TusA, Mozia - VI. Rapporto preliminare della Missione congiunta con la Soprintendenza alle Antichità della Sicilia Occidentale (Studi Semitici 37), Roma 1970, pp. 51-62.

1984 La scultura in pietra di Selinunte, Palermo 1984.

WHITAKER, J.I.S.

1921 Motya: a Phoenician colony in Sicily, London 1921.

WINTER, F.

1903 Die antiken Terrakotten. Die Typen der figürlichen Terrakotten, III, 1-2, Berlin 1903. 

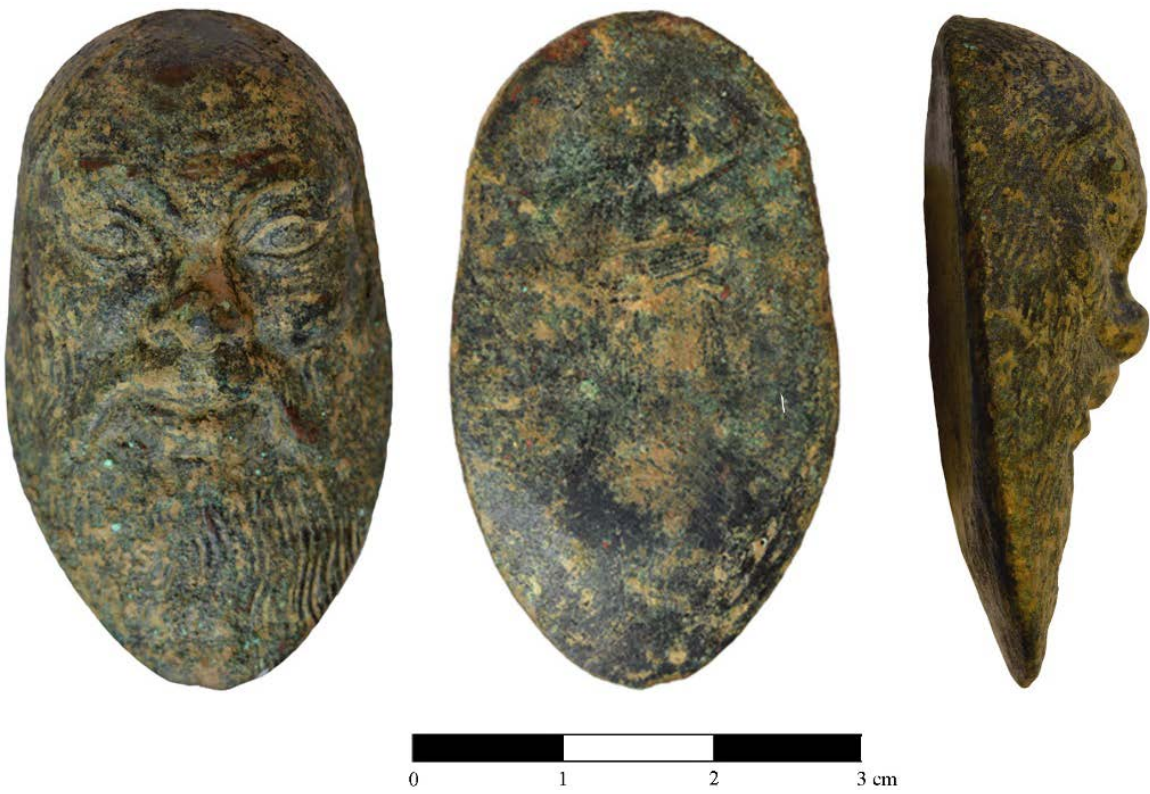

Fig. 1 - Testa di sileno in bronzo MAC.15.500.



Fig. 2 - Disegno della testa in bronzo MAC.15.500 (disegno A. Sumislawska). 




Fig. 3 - Disegno della sezione della testa in bronzo MAC.15.500.

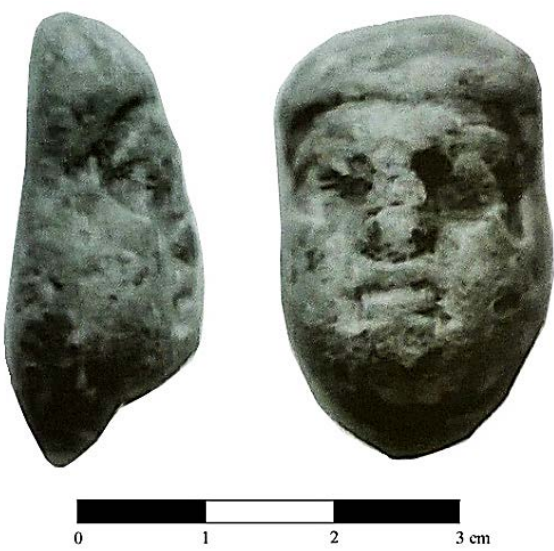

Fig. 4 - Peso in piombo configurato a testa di sileno da Amrit (da Elayi - Elayi 1997, tav. XXXVI). 


\section{SOMMARIO}

DipartimENTo SCIENZE DELl'ANTICHITÀ

SEZIONE di ORIENTALISTICA

\section{VICINO ORIENTE \\ XIX - 2015}

L. Nigro - Bethlehem in the Bronze and lron Ages
in the light of recent discoveries by the Palestinian MOTA-DACH

$\checkmark$. Pisaniello - Parallel passages among Hittite-Luwian rituals:

for the restoration of $K U B 35.146$

F. Spagnoli - Una testa di sileno in bronzo da Mozia

N. Chiaren

dall Area sacra del Kothon a Mozia

G. Labisi - al-Fudayn: an Umayyad residence in Northern Jordan

P. Buzi - Early Christianity in the Fayyūm: the new contribution of archaeology

I. Materia - Preliminary notes on the ware depicted on the ceiling

of the Cappella Palatina in Palermo

S. Autiero - Indian Ocean trade:

a reassessment of the pottery find
$\left(3^{\text {rd }}\right.$ century $B C-S^{S^{t h}}$ century $\left.A D\right)$

M.M. Jamhawi - N. Al-Shakarchi - I. Al-Hashimi

Assessment of tourists' satisfaction in the downtown of Amman

SCAVI E RICERCHE

L. Nigro - C. Fiaccavento - M. Jaradat - J. Yasine

A

L. Nigro - D. Montanari - M. Ghayyada - J. Yasine

A Middle Bronze and Iron Age necropolis near Bethlehem (Palestine)

L. Nigro - G. Ripepi - I. Hamdan - J. Yasine

15 Interim Report

and valorization of archaeological heritage

R. Francia - L'archivio di tavolette del complesso B-C-H di Büyükkale

organizazione degli archivi reali ittiti. Considerazioni preliminari

V. Pisaniello - La collezione di tavolette del complesso B-C-H di Büyükkale

T. De Vincenzi - L'archivio di tavolette del complesso B-C-H
sull'acropoli di Büyükkale

Museo del Vicino Oriente, Egitto e Mediterraneo

L. Nigro - Il nuovo allestimento del Museo del Vicino Oriente,

Egitto e Mediterraneo della Sapienza

D. Montanari - Bollettino delle attività del Museo del Vicino Oriente,

Egito e Meditraneo della Sapienza, anno 2015

RECENSIONI

A. Orsingher - E. PAPPA (2013), Early Iron Age Exchange in the West:

(Ancient Near Eastern Studies Supplement Series 43)

Leuven - Paris - Walpole 2013, MA.: Peeters $\frac{1}{2}$

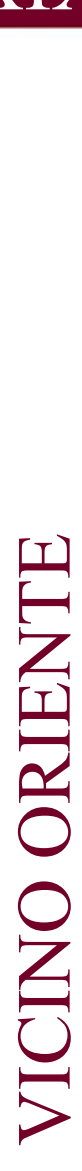

345

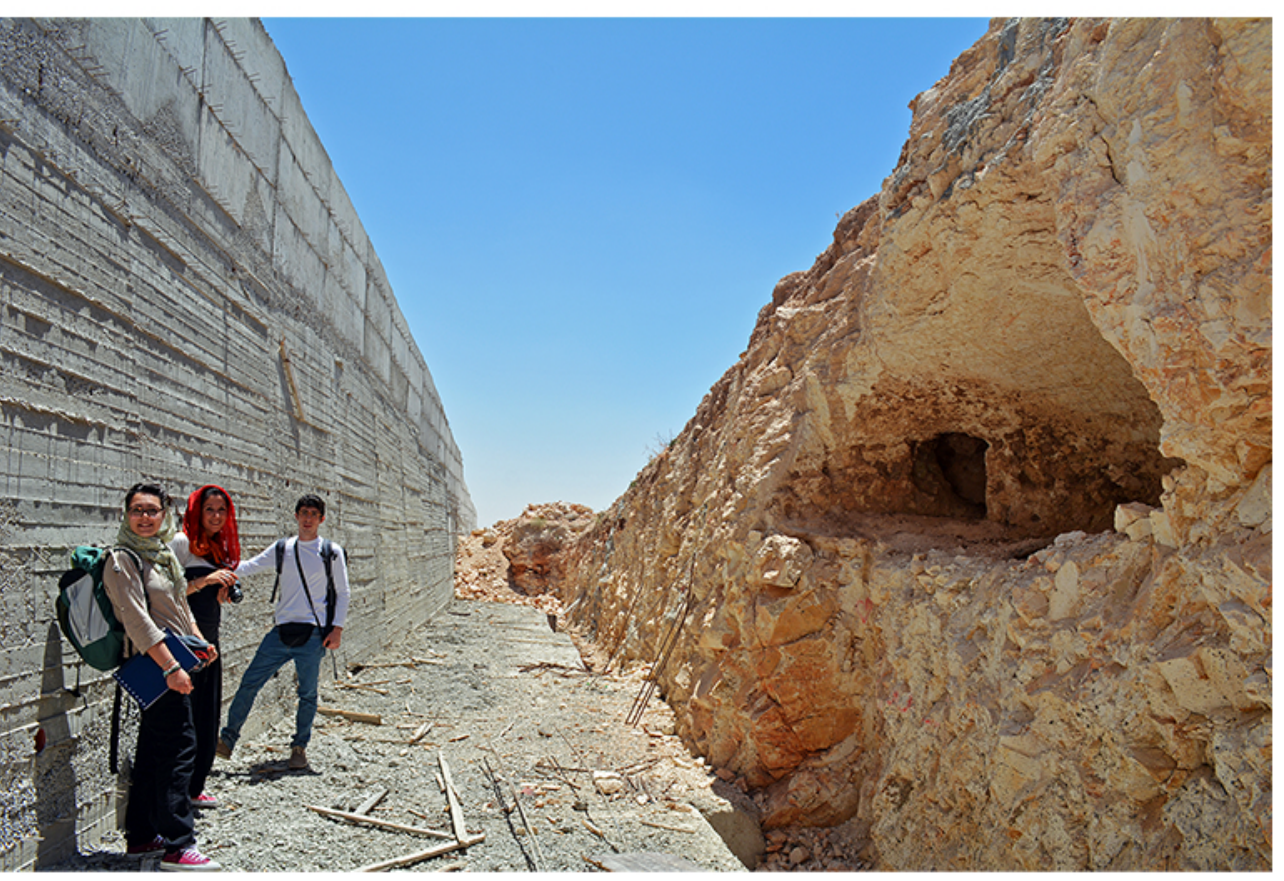

ROMA 2015 


\section{VICINO ORIENTE XIX - 2015}


VICINO ORIENTE

SAPIENZA UNIVERSITÀ DI ROMA

DIPARTIMENTO SCIENZE DELL'ANTICHITÀ

SEZIONE DI ORIENTALISTICA

Scientific Editor: Lorenzo Nigro

International Scientific Committee: Brian Rose, Frank Braemer, Mounir Fantar, Piero Bartoloni, Thomas Schaefer, Zeidan Kafafi

National Scientific Committee: Carlo Giovanni Cereti, Maria Vittoria Fontana, Sebastiano Tusa, Massimiliano Marazzi

Editorial Board: Daria Montanari, Chiara Fiaccavento

Tipografia: SK7 - Roma

ISSN 0393-0300

Rivista con comitato di referee

Journal with international referee system

www.lasapienzatojericho.it/SitoRivista/Journal/Rivista.php

In copertina: Tomba B9, necropoli di Khalet al-Jam’a (Betlemme). 
VICINO ORIENTE

SAPIENZA UNIVERSITÀ DI ROMA

DIPARTIMENTO SCIENZE DELL'ANTICHITÀ

SEZIONE DI ORIENTALISTICA

\section{SOMMARIO}

ARTICOLI

L. Nigro - Bethlehem in the Bronze and Iron Ages

in the light of recent discoveries by the Palestinian MOTA-DACH

V. Pisaniello - Parallel passages among Hittite-Luwian rituals:

for the restoration of KUB 35.146

F. Spagnoli - Una testa di sileno in bronzo da Mozia

N. Chiarenza - Una matrice per terrecotte con sileno dall'Area sacra del Kothon a Mozia

G. Labisi - al-Fudayn: an Umayyad residence in Northern Jordan

P. Buzi - Early Christianity in the Fayyūm: the new contribution of archaeology

I. Materia - Preliminary notes on the ware depicted on the ceiling

of the Cappella Palatina in Palermo

S. Autiero - Indian Ocean trade:

a reassessment of the pottery finds from a multidisciplinary point of view ( $3^{\text {rd }}$ century $B C-5^{\text {th }}$ century $\left.A D\right)$

M.M. Jamhawi - N. Al-Shakarchi - I. Al-Hashimi

Assessment of tourists' satisfaction in the downtown of Amman

SCAVI E RICERCHE

L. Nigro - C. Fiaccavento - M. Jaradat - J. Yasine Archaeology from A to Z: Abu Zarad, an ancient town in the heartland of Palestine

L. Nigro - D. Montanari - M. Ghayyada - J. Yasine

Khalet al-Jam'a. A Middle Bronze and Iron Age necropolis near Bethlehem (Palestine) 185 
VICINO ORIENTE

SAPIENZA UNIVERSITÀ DI ROMA

DIPARTIMENTO SCIENZE DELL'ANTICHITÀ

SEZIONE DI ORIENTALISTICA

L. Nigro - G. Ripepi - I. Hamdan - J. Yasine

The Jericho Oasis Archaeological Park - 2015 Interim Report.

Italian-Palestinian Cooperation for protection

and valorization of archaeological heritage

R. Francia - L'archivio di tavolette del complesso B-C-H di Büyükkale

e l'organizzazione degli archivi reali ittiti. Considerazioni preliminari

V. Pisaniello - La collezione di tavolette del complesso B-C-H di Büyükkale

T. De Vincenzi - L'archivio di tavolette del complesso B-C-H sull'acropoli di Büyükkale

Museo del Vicino ORIente, Egitto e MediterRaneo

L. Nigro - Il nuovo allestimento del Museo del Vicino Oriente,

Egitto e Mediterraneo della Sapienza

D. Montanari - Bollettino delle attività del Museo del Vicino Oriente,

Egitto e Mediterraneo della Sapienza, anno 2015

\section{RECENSIONI}

A. Orsingher - E. PAPPA (2013), Early Iron Age Exchange in the West:

Phoenicians in the Mediterranean and the Atlantic

(Ancient Near Eastern Studies Supplement Series 43),

Leuven - Paris - Walpole 2013, MA.: Peeters 